\title{
C-Jun drives melanoma progression in PTEN wild type melanoma cells
}

\author{
Melanie Kappelmann-Fenzl ${ }^{1,2}$, Claudia Gebhard ${ }^{3,4}$, Alexander O. Matthies ${ }^{1}$, Silke Kuphal ${ }^{1}$, Michael Rehli ${ }^{3,4}$ and \\ Anja Katrin Bosserhoff,
}

\begin{abstract}
Due to the critical impact of active AP-1 transcription factors in melanoma, it is important to define their target genes and to identify and ultimately inhibit oncogenic signals. Here we mapped the genome-wide occupancy of the AP-1 family member c-Jun in different melanoma cells and correlated AP-1 binding with transcriptome data to detect genes in melanoma regulated by $c-J u n$. Our analysis shows that $c$-Jun supports the malignant phenotype by deregulating genes in cancer-relevant signaling pathways, such as mitogen-activated protein kinase (MAPK) and phosphatidylinositol-3-kinase (PI3K) pathways. Moreover, we demonstrate that the importance of $c$-Jun depends on melanoma stage and mutation status of the tumor suppressor PTEN. Our study reveals that activation of $c$-Jun overrules the tumor suppressive effect of PTEN in early melanoma development. These findings help to understand the relevance of $c$-Jun within cancer pathways in different melanoma cell types, especially in relation to MAPK and PI3K pathways, which are commonly deregulated in melanomas. Consequently, targeting c-Jun in PTEN ${ }^{+}$melanoma cells may represent a promising therapeutic strategy to inhibit survival of melanoma cells to prevent the development of a metastatic phenotype.
\end{abstract}

\section{Introduction}

Melanoma is a highly aggressive and heterogeneous type of cancer, and its incidence is growing faster than any other cancer entity. In recent decades, many altered pathways regulating the development and progression of melanoma and the high migratory and invasive potential of melanoma cells have been identified, but a detailed molecular understanding of this disease is largely lacking.

One crucial step in melanoma development and progression is the deregulation of cancer-supporting transcription factors, especially activating protein-1 (AP-1) transcription factors, including the $c$-Jun, JDP, c-Fos, FRA, and $M A F$ subfamilies $^{1}$. AP-1 proteins bind to the classical palindromic recognition sequence $5^{\prime}-\mathrm{TGA}(\mathrm{C} / \mathrm{G}) \mathrm{TCA}-3^{\prime}$ and regulate target gene expression, leading to

\footnotetext{
Correspondence: Anja Katrin Bosserhoff (anja.bosserhoff@fau.de)

${ }^{1}$ Institute of Biochemistry (Emil-Fischer Center), Friedrich-Alexander University

Erlangen-Nürnberg, Erlangen, Germany

${ }^{2}$ Faculty of Applied Health Care Sciences, University of Applied Science,

Deggendorf, Germany

Full list of author information is available at the end of the article.

Edited by Z.-X. Xiao
}

deregulation of cancer-relevant signaling pathways. Thus, AP-1 transcription factor dimers play an important role in different cancer types, including malignant melanoma ${ }^{1-5}$. A main characteristic of AP-1 complexes in the cell is their heterogeneity in dimer composition. This heterogeneity is caused by the fact that multiple AP-1 subunits can be expressed simultaneously, and different dimer compositions lead to the transcriptional regulation of different target genes. Various studies demonstrated that differences in AP-1 dimer compositions cause altered specificity in their binding site selection ${ }^{6}$. However, it remains unclear which direct target genes of AP-1 homodimer or heterodimer cause the functional effects that support melanomagenesis.

The AP-1 family member $c$-Jun is a main regulator of melanoma progression ${ }^{4,7,8}$, acts by regulating target genes supporting proliferation and migration of cancer cells, and thus promotes the malignant phenotype. We have previously demonstrated that the microRNA miR-125b directly regulates the transcription factor $c$-Jun, affecting the proliferative and migratory potential of melanoma

\section{(c) The Author(s) 2019}

(c) (i) Open Access This article is licensed under a Creative Commons Attribution 4.0 International License, which permits use, sharing, adaptation, distribution and reproduction c. in any medium or format, as long as you give appropriate credit to the original author(s) and the source, provide a link to the Creative Commons license, and indicate if changes were made. The images or other third party material in this article are included in the article's Creative Commons license, unless indicated otherwise in a credit line to the material. If material is not included in the article's Creative Commons license and your intended use is not permitted by statutory regulation or exceeds the permitted use, you will need to obtain permission directly from the copyright holder. To view a copy of this license, visit http://creativecommons.org/licenses/by/4.0/. 
cells ${ }^{9}$. Furthermore, we identified an alternative regulatory pathway of $c-J u n$ in melanoma cells leading to upregulation of $c$-Jun activity via the loss of the cell-adhesion molecule E-cadherin ${ }^{10,11}$. Despite its role in the aforementioned pathophysiological processes, only a few specific c-Jun target genes have been identified to date. Previous chromatin immunoprecipitation (ChIP) studies focusing on AP-1/c-Jun in nonmelanoma cell types identified a few cancer-related target genes of c-Jun. Deregulation of $c$-Jun is one of the most important events in malignant melanoma and many other cancer entities, but the functional relevance of $c$-Jun deregulation and its molecular effects on target gene expression have not been determined in detail to date.

Another crucial event supporting cancer cell survival is the upregulation of PI3K/AKT signaling activity in various cancer types, which can largely be attributed to deregulation of the $A K T$ negative regulator, phosphatase, and tensin homolog (PTEN) phosphatase ${ }^{12}$. Mutations in the PTEN gene and thus the loss of this tumor suppressor protein are prevalent in melanoma and lead to upregulation of $A K T$ activity. Activated $A K T$ protects cells from apoptosis by phosphorylating and inactivating proapoptotic substrates ${ }^{13}$. Mutational inactivation of PTEN frequently occurs in human cancers; however, early molecular tumor-promoting mechanisms in PTEN expressing $\left(P T E N^{+}\right)$cancer cells, such as those in early developing melanoma, remain unclear.

In this study, we demonstrate that c-Jun upregulation in melanoma cells depends on melanoma stages (primary tumor (PT) vs. metastasis (MET)) and PTEN expression status.

\section{Results}

\section{C-Jun and the tumor suppressor PTEN exhibit a positive} correlation in melanoma cells

Given that melanomas gain different properties during their progression, we selected six different melanoma cell lines (Sbcl-2, WM3211, WM793, WM1366, WM1158, WM9) categorized by tumor stage (PT, MET) and their previously described BRAF and PTEN mutation status ${ }^{14}$. All cell line characteristics are shown in Supplementary Table 1, and the described mutations of each cell line were confirmed by our RNA sequencing data (Supplementary Table 1, Supplementary Fig. 2). We first performed functional assays to analyze the proliferative (Supplementary Fig. 1Ai) and/or migratory potential (Supplementary Fig. 1Aii) of four melanoma cell lines exhibiting different tumor stages, BRAF mutation statuses, and PTEN deletions. Interestingly, based on the mentioned characteristics, a distinct classification based on proliferative and/or migratory behavior was not possible. Thus, we further focused on c-Jun-dependent differences of gene expression among the analyzed melanoma cell lines.
We first analyzed differentially expressed genes in malignant melanoma by performing RNA-Sequencing (RNA-seq) with all aforementioned melanoma cell lines and normal human epidermal melanocytes (NHEM). We assigned tumor stage and mutation status to our samples and performed principal component analyses (PCA) to construct low-dimensional representations of the expression profiles of the complete RNA-Seq data set. The PCA results clearly demonstrated sample clustering based on PTEN and BRAF status (Fig. 1a).

Analysis of mRNA expression levels of both c-Jun and PTEN in all used melanoma cell lines (compared to NHEMs) revealed high c-Jun expression levels in $P T E N^{\mathrm{WT}}$ cells that were gradually reduced in $P T E N^{\mathrm{HemDel}}$ and PTEN ${ }^{\text {HomDel }}$ cells (Fig. 1b). PTEN mRNA expression follows the corresponding copy numbers of the used cell lines, demonstrating high PTEN mRNA levels in NHEMs and $P T E N^{\mathrm{WT}}$ that continuously decrease in $P T E N^{\mathrm{HemDel}}$ and $P T E N^{\text {HomDel }}$ (Fig. 1c). We exemplarily confirmed $c$ Jun and PTEN mRNA expression via qRT-PCR analysis in one $P T E N^{\mathrm{WT}}$ (WM1366) and one PTEN ${ }^{\mathrm{HomDel}}$ (WM9) melanoma cell line (Fig. 1d, e) compared to NHEMs. In accordance with these findings, Western blot analyses of $P T E N^{\mathrm{WT}}$ melanoma cells exhibited high $c$-Jun protein levels, which decreased with the gradual loss of PTEN (Fig. 1f, Supplementary Fig. 1B). Due to the fact that $P T E N$ is known to inhibit $A K T$ activity (predominantly $A K T 3$ in melanoma) and thus promotes melanoma cell apoptosis, loss of PTEN is a crucial event during melanoma development and progression. Hence, we further analyzed $A K T 1$ and $A K T 3$ mRNA expression by qRTPCR in both $P T E N^{+}$and $P T E N^{-}$melanoma cells. Interestingly, no differences in $A K T$ expression between $P T E N^{\mathrm{WT}}$ and $P T E N^{\mathrm{HomDel}}$ cells were detected (Fig. 1g), supporting the role of loss of PTEN on AKT activity.

Consequently, we hypothesize that $P T E N^{+}$melanoma cells in early melanoma development utilize c-Jun to overcome the tumor-suppressive effect of PTEN.

\section{C-Jun significantly influences gene expression in malignant melanoma}

To further explore the $c$-Jun influences gene expression in malignant melanoma, we mapped its binding sites using ChIP-Sequencing (ChIP-seq). First, we identified genes associated with $c$-Jun peaks in each melanoma cell line and compared their mRNA expression to normal NHEMs. Our analysis showed a significant induction of $c$ Jun peak-associated genes in each sequenced c-Jun expressing melanoma cell line (Sbcl2, WM3211, WM1366, WM793, and WM1158), suggesting a positive regulatory activity of $c$-Jun (Fig. 2a). Moreover, analysis of the resulting c-Jun ChIP-Seq peaks confirmed the melanoma cell line clustering detected via RNA-Seq (Fig. 1a). Most interestingly, our c-Jun ChIP-Seq data clearly 
A

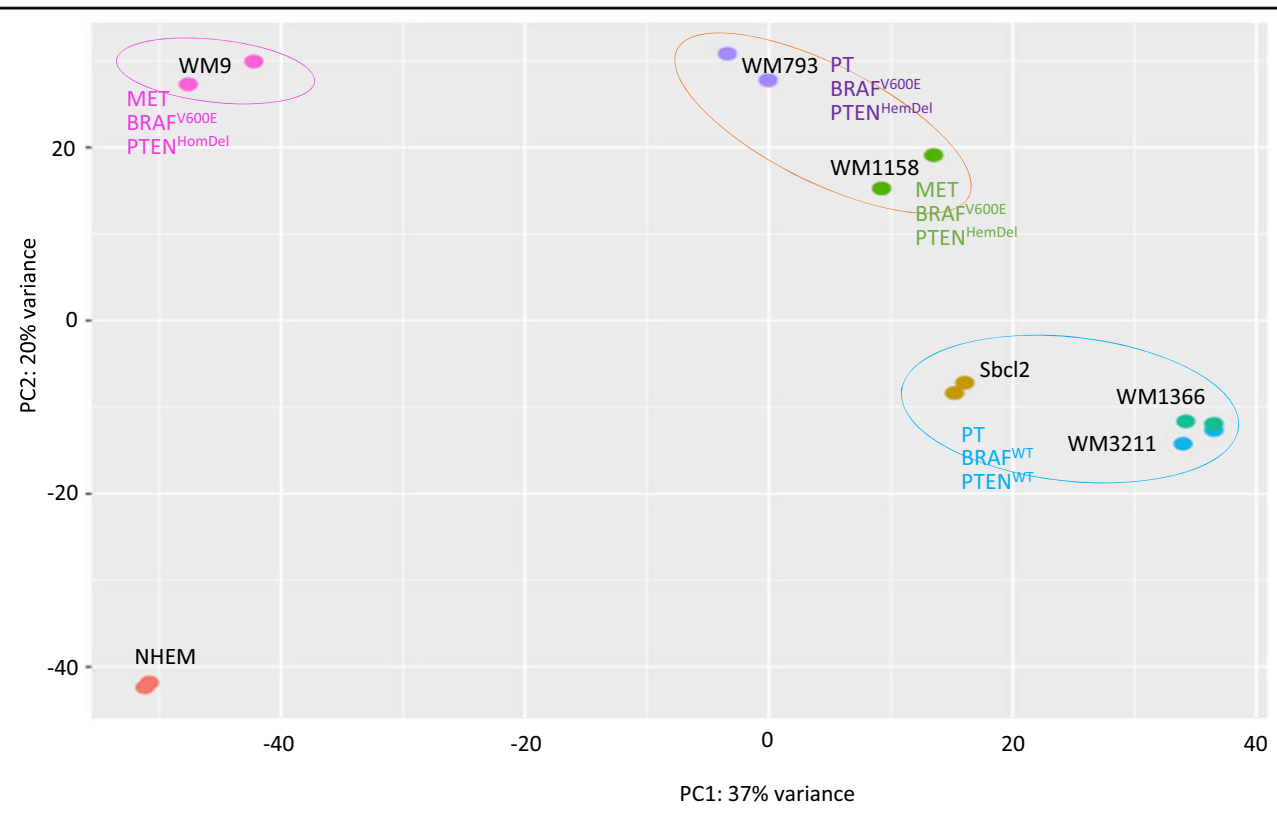

B

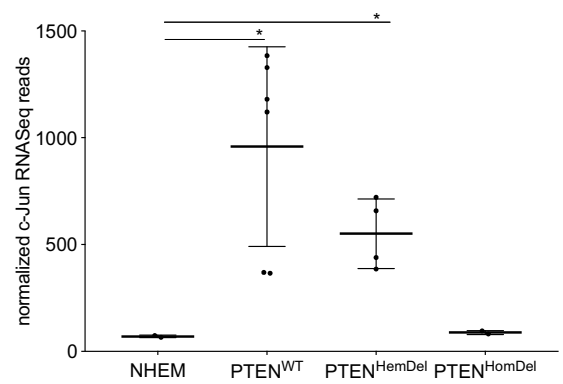

D

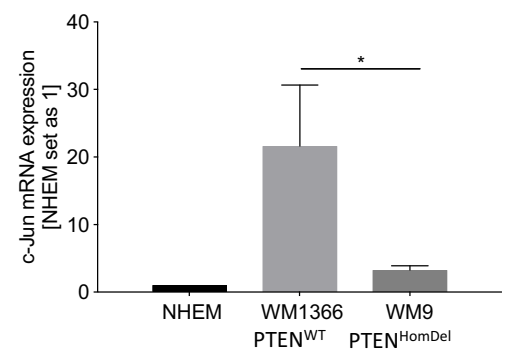

F
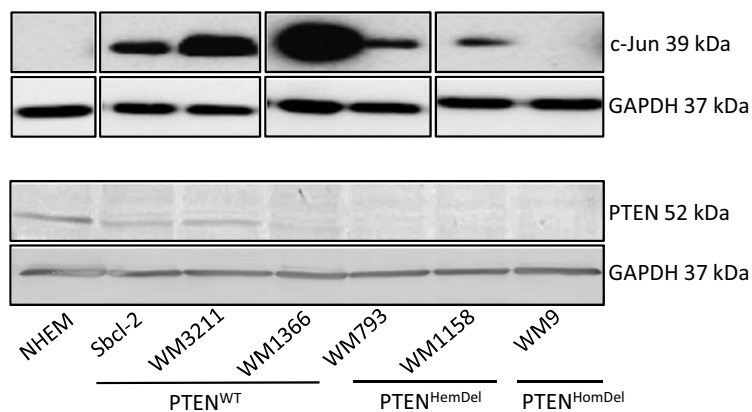

C

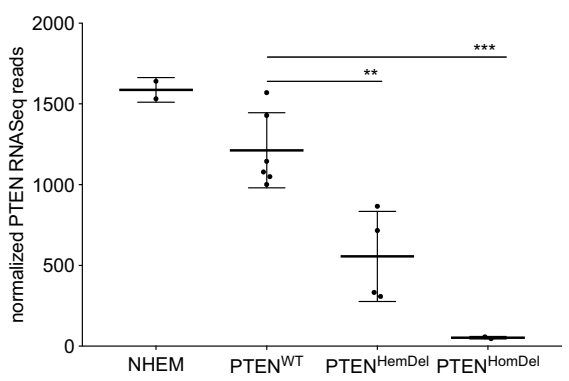

E

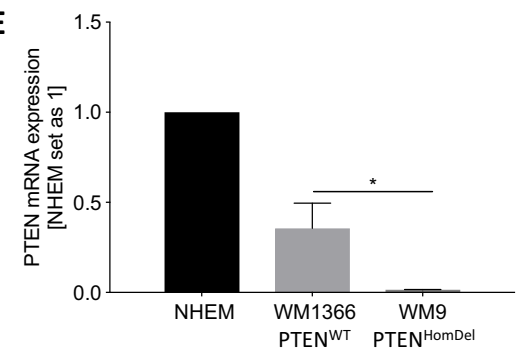

G

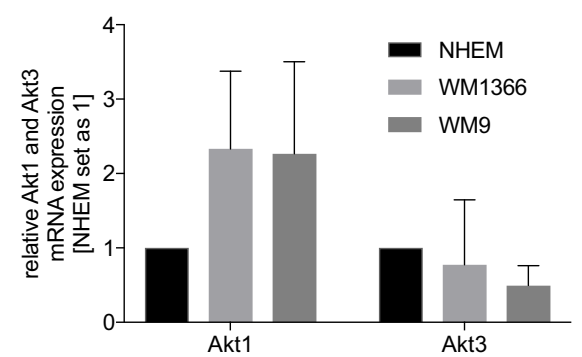

Fig. 1 (See legend on next page.) 
(see figure on previous page)

Fig. 1 C-Jun and PTEN exhibit a positive correlation in malignant melanoma. a Low-dimensional representations of the expression profiles of the RNA-Seq data (NHEM, Sbcl2, WM3211, WM1366, WM793, WM1158, WM9) by principal component analyses (PCA) (DESeq2 in R, Bioconductor). RNA-Seq data visualization revealed sample clustering based on PTEN and BRAF expression status. b Normalized c-Jun RNA-Seq reads. c Normalized PTEN RNA-Seq reads. $\mathbf{d}$ c-Jun mRNA expression analysis via qRT-PCR normalized to ß-actin. e PTEN mRNA expression analysis via qRT-PCR normalized to $B$-actin. $\mathbf{f}$ Western blot analysis revealing c-Jun and PTEN protein levels in different melanoma cell lines compared with NHEMs. C-Jun Western blot positions are adapted and the original Western Blot is shown in Supplementary Fig. 1B. GAPDH serves as loading control. g AKT1 and AKT3 mRNA expression analysis via qRT-PCR normalized to $B$-actin. The box-plots show the mean \pm s.d. of read counts of two independent experiments ${ }^{* * *} P<$ $\left.0.001 ;{ }^{*} P<0.01 ;{ }^{*} P<0.5\right)$. The bars show the mean \pm s.d. of three independent experiments; measurements were performed in triplicate $\left({ }^{*} P<0.5\right)$

revealed differences in DNA-binding activity of c-Jun based on the cell line-specific PTEN copy number alteration. Grouping of $c$-Jun peak sets by PTEN expression status resulted in 17.953 common $c$-Jun peaks in PTEN $^{\mathrm{WT}}$ (Sbcl-2, WM3211, WM1366) and 13.801 in PTEN $^{\text {HemDel }}$ (WM793, WM1158) cell lines (Fig. 2b). The abundance and overlap of c-Jun peaks across melanoma cells confirm the relationship between c-Jun activity and PTEN expression status as observed on transcript level.

To further study the impact of c-Jun on the activity status of bound regulatory elements, we compared the local deposition of H3K27ac, a histone modification associated with active regulatory sites, at c-Jun peaks (Supplementary Table 2) in melanoma cells compared to NHEMs. As shown in heatmap representations and corresponding histograms, c-Jun-bound regions show a higher local acetylation in melanoma compared to melanocytes (Fig. 2c).

Taken together, our data demonstrate that gene expression differences between all analyzed melanoma cell lines depend on c-JUn and PTEN expression/activity. These findings indicate that $c$-Jun plays a crucial role in early melanoma development.

\section{Functional annotation reveals an enrichment of PI3K/AKT signaling in PTEN $^{+} / c-J$ In $^{+}$melanoma cells}

As a next step, we were interested in the functional relevance of all differentially expressed genes (based on RNA-Seq) and the subset of differentially expressed genes associated with a $c$-Jun peak. We performed differential expression analysis of the RNA-Seq data described above and compared the gene expression status of $P T E N^{\mathrm{WT}}$, $P T E N^{\text {HemDel }}$, or PTEN ${ }^{\text {HomDel }}$ cells with NHEMs. The comparison resulted in three different gene sets with differentially expressed genes represented as heat maps (Fig. 3a, total diffgenes). These gene sets were subsequently merged with c-Jun ChIP-Seq peak annotation data to identify those genes differentially expressed, which are regulated by c-Jun (Fig. 3b, diffgenes Jun). The amount of total differentially expressed genes and differentially expressed genes associated with a c-Jun peak of all generated gene sets are illustrated in Supplementary Table 3. Regarding PTEN ${ }^{\text {HomDel }}$ cells (lacking $c$-Jun), we further explored the total differentially expressed genes.
These newly generated data sets, including the total differentially expressed genes and differentially expressed genes associated with a $c$-Jun peak, were used to perform detailed functional annotation clustering with the bioinformatic tools Database for Annotation, Visualization and Integrated Discovery (DAVID; v 6.8) and Gene Set Enrichment Analysis (GSEA) tool, respectively, to identify enriched gene sets within each data set and their molecular functions.

Functional annotation analysis via DAVID ${ }^{15,16}$ resulted in several melanoma-/cancer-specific enriched Kyoto Encyclopedia of Genes and Genomes (KEGG) pathways (Table 1). Interestingly, functional annotation of the differentially expressed genes associated with a c-Jun peak yielded similar enriched pathways in $P T E N^{\mathrm{WT}}$ and $P T E N^{\mathrm{HemDel}}$ cells as the total differentially expressed genes of PTEN ${ }^{\mathrm{HomDel}}$ cells. Our analysis revealed c-Jun to be a regulator of genes involved in the PI3K/AKT signaling pathway in $P T E N^{+}$melanoma cells, indicating that c-Jun mainly supports anti-apoptotic and pro-survival processes in early $\left(P T E N^{\mathrm{WT}}\right.$ and $\left.P T E N^{\mathrm{HemDel}}\right)$ melanoma development.

The GSEA tool utilizes the expression status (read counts of RNA-Seq) for functional annotation clustering and thus identifies genes that are upregulated or downregulated. GSEA results of our RNA-Seq data are depicted as enrichment plots and the corresponding Blue-Pink O' Gram in the Space of the Analyzed GeneSets of Pathways in cancer of all five comparisons (NHEM vs. PTEN ${ }^{\mathrm{WT}}, P T E N^{W T}{ }^{\mathrm{T}}$-Jun, $P T E N^{\text {HemDel }}, P T E N^{\text {HemDel }}$-Jun, or PTEN ${ }^{\text {HomDel }}$, Supplementary Fig. 3). The outcome is represented in Profile of the Running ES Score \& Positions of GeneSet Members on the Rank Ordered List. Similar to our previous functional annotation results, we observed $c$-Jun-dependent regulation of PI3K/AKT signaling members in $P T E N^{+}$melanoma cells (Supplementary Fig. 3; red arrows).

As a next step, we assigned biological meaning to the $c$ Jun ChIP-Seq peaks in noncoding genomic regions analyzing the peak annotations by the Genomic Regions Enrichment of Annotations Tool (GREAT), a bioinformatic tool that predicts functions of cis-regulatory regions. We used $c$-Jun peaks of genomic regions detected by $c$-Jun ChIP-Seq in PTEN ${ }^{\mathrm{WT}}$ and PTEN ${ }^{\text {HemDel }}$ cells. GREAT analysis resulted in an enrichment of several 


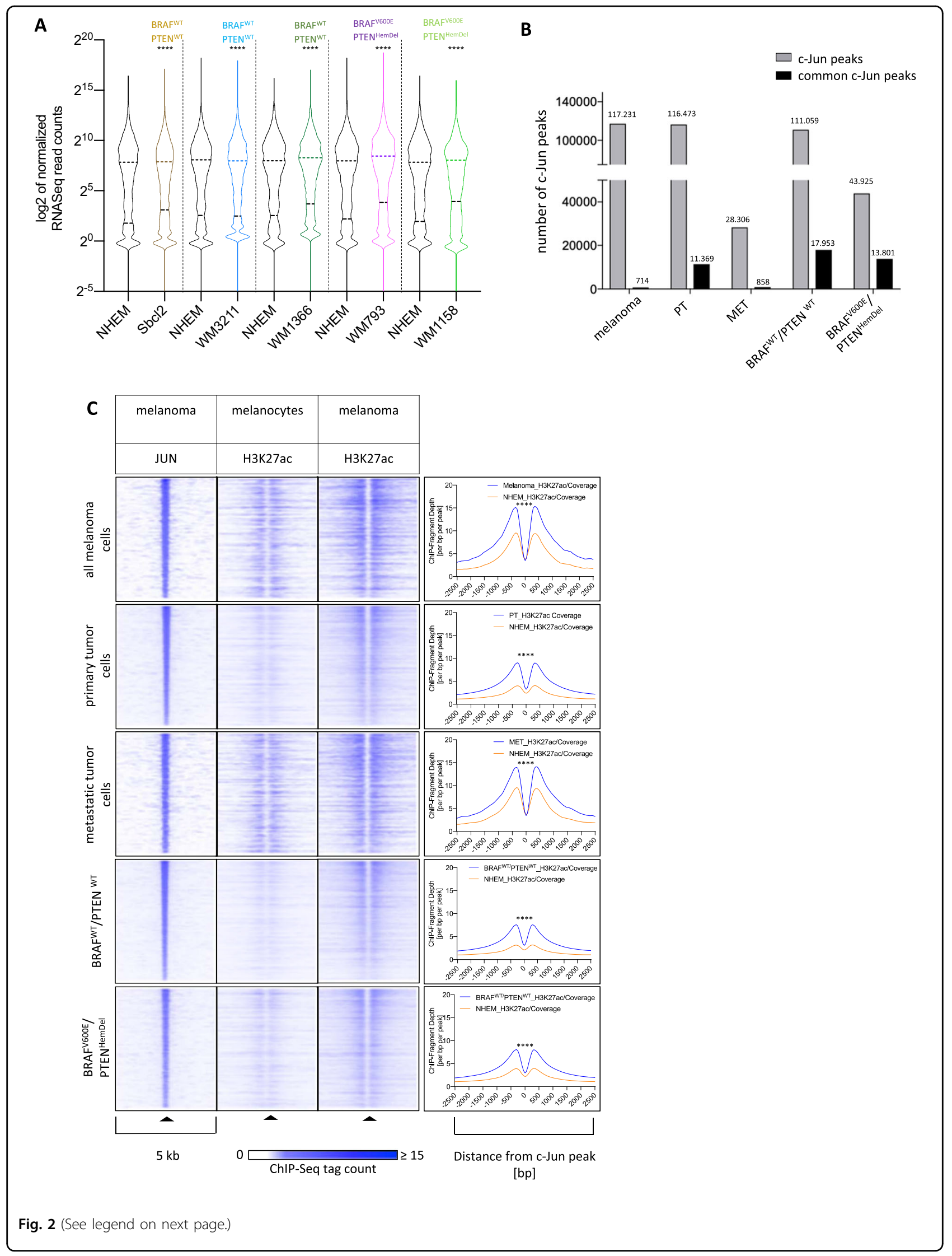


(see figure on previous page)

Fig. 2 C-Jun significantly influences gene expression alteration in malignant melanoma. a All sequenced melanoma cell lines show significant differences in gene expression due to the regulatory activity of c-Jun compared to NHEMs. Violin blots display the interquartile ranges (25-75\%) with an intersection as the median; Significantly different distributions in pairwise comparisons are indicated (**** $P<0.0001$, Wilcoxon test, two-sided). b Clustering of c-Jun peak sets by PTEN expression status revealed a correlation between c-Jun activity and PTEN expression. c Heat maps and histograms of ChIP-Seq tag counts of the histone acetylation status (H3K27ac) in a 5-kb wide range around c-Jun peaks. Regions centered on c-Junbound regions were clustered according to their H3K27ac ChIP-Seq profiles in melanoma and NHEMs, illustrating a high acetylation rate around the genomic $c$-Jun peaks in melanoma and a low rate in NHEMs (***P $<0.0001$, Wilcoxon test, paired, two-sided)
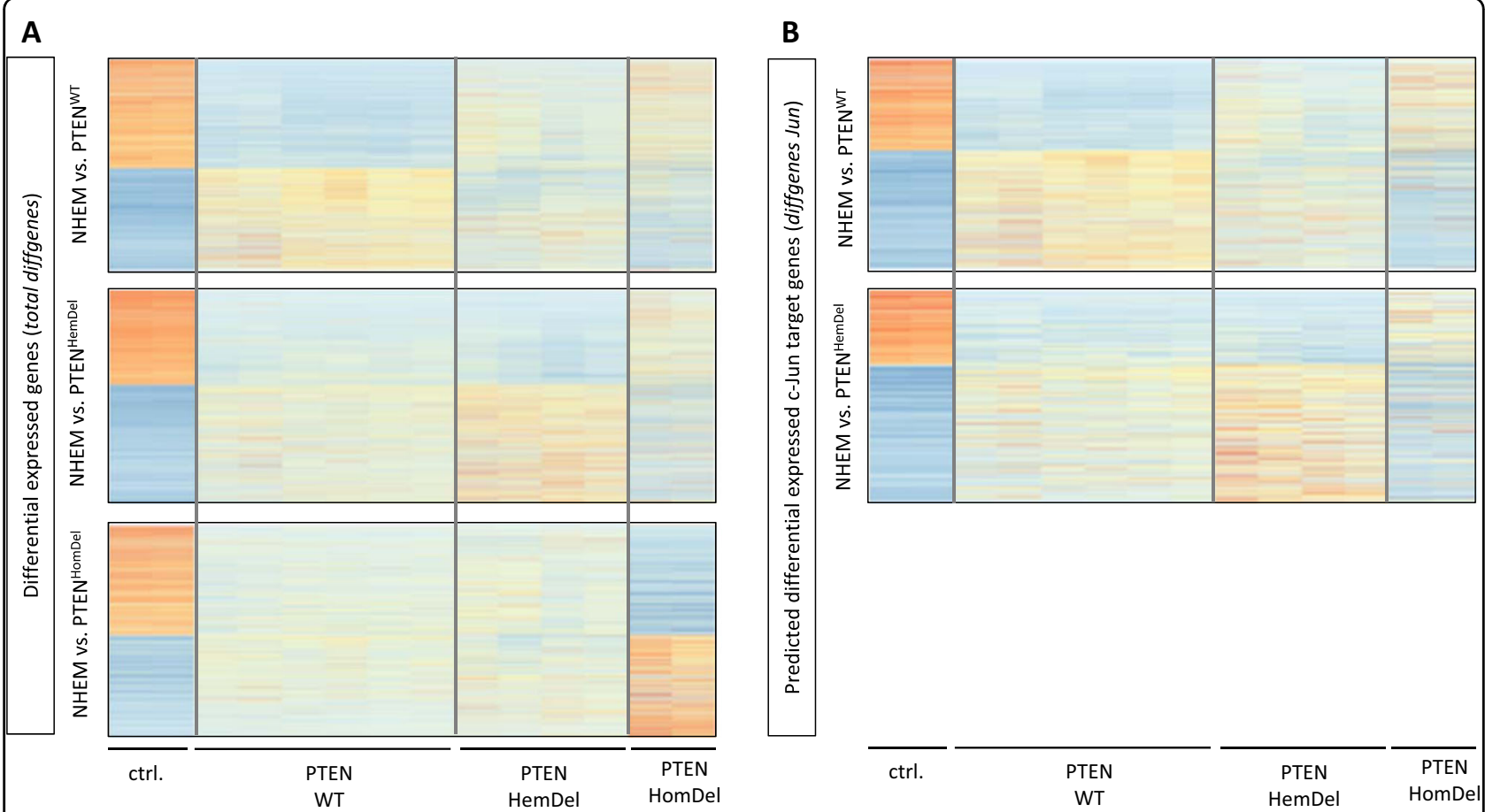

Fig. 3 Gene set enrichment analysis resulted in an enrichment of PI3K/AKT signaling in PTEN-expressing and c-Jun-expressing melanoma cells. a Differential expression analysis via DESeq2 (R, Bioconductor). Heat maps illustrate RNA-Seq differential expression in PTEN ${ }^{\text {WT }}$, PTEN ${ }^{\text {HemDel }}$, or PTEN ${ }^{\text {HomDel }}$ cells compared to NHEMs. b RNA-Seq differential expression results combined with c-Jun ChIP-Seq peaks illustrated as heat maps of differentially expressed genes regulated by c-Jun in PTEN ${ }^{W T}$ and PTEN ${ }^{\text {HemDel }}$ cells

different cancer-relevant pathways, such as PI3K/AKT in both melanoma subgroups PTEN ${ }^{\mathrm{WT}}$ and PTEN ${ }^{\mathrm{HemDel}}$, confirming our prior findings.

Furthermore, the identified gene associations of the $c$ Jun ChIP-Seq peaks of PTEN ${ }^{\mathrm{WT}}$ and PTEN ${ }^{\mathrm{HemDel}}$ cells were adjusted to the corresponding RNA-Seq differential expression results. These analyses revealed a deregulation of 1084 c-Jun-associated genes in PTEN ${ }^{\mathrm{WT}}$ cells (among overall 4188 differentially expressed genes in $P T E N^{\mathrm{WT}}$ compared to NHEMs) and 736 (among overall 2764 differentially expressed genes in $P T E N^{\text {HemDel }}$ compared to NHEMs) in PTEN ${ }^{\text {HemDel }}$ cells (Supplementary Table 3, diffgenes c-Jun). Functional network analyses via STRING 10.5 (Search Tool for the Retrieval of Interacting Genes/ Proteins) of both gene sets, including genes regulated by c-Jun in PTEN ${ }^{\mathrm{WT}}$ and PTEN ${ }^{\mathrm{HemDel}}$, respectively, also revealed an enrichment of the KEGG pathways similar to those identified by DAVID, GSEA, and GREAT. The number of gene set members and false discovery rates are presented in Supplementary Fig. 4.

In summary, numerous differentially expressed genes in $P T E N^{\mathrm{WT}}$ and $P T E N^{\mathrm{HemDel}}$ cells belonging to the PI3K/ AKT-signaling pathway are regulated by c-Jun (Supplementary Fig. 5, STRING; red: members of PI3K-signaling system), indicating again that $c$-Jun plays a crucial role in early melanoma development to promote survival in the presence of PTEN.

\section{C-Jun directly regulates PI3K/AKT members in PTEN- positive melanoma cells}

Next, we confirmed the identified c-Jun-regulated PI3K/ AKT-related targets by visualizing c-Jun and H3K27 ChIP-Seq peaks and the corresponding RNA-Seq expression data in the IGV Browser ${ }^{17}$. We identified 
Table 1 DAVID functional annotation analysis. Functional annotation analysis of RNA-Seq data via DAVID of the five generated gene sets (NHEM vs. PTEN ${ }^{\mathrm{WT}}$, PTEN $^{\text {HemDel, }}$ PTEN $^{\text {HomDel }}$, PTEN ${ }^{\text {WT }}$ C-Jun or PTEN ${ }^{\text {HemDel }}$ C-Jun) depicting similar results in terms of enriched KEGG pathways

\begin{tabular}{|c|c|c|}
\hline \multicolumn{3}{|l|}{ total diffgenes } \\
\hline KEGG pathway & Size & $P$-value \\
\hline \multicolumn{3}{|l|}{ NHEM-PTEN ${ }^{W T}$} \\
\hline Pathways in cancer & 114 & $3.23 \mathrm{E}-05$ \\
\hline PI3K-Akt signaling pathway & 90 & 7.94E-03 \\
\hline Rap1 signaling pathway & 71 & $6.23 \mathrm{E}-06$ \\
\hline Focal adhesion & 68 & 2.43E-05 \\
\hline Endocytosis & 68 & $1.69 \mathrm{E}-02$ \\
\hline Proteoglycans in cancer & 67 & $1.66 \mathrm{E}-05$ \\
\hline Ras signaling pathway & 62 & $9.68 \mathrm{E}-03$ \\
\hline Lysosome & 61 & $5.40 \mathrm{E}-13$ \\
\hline \multicolumn{3}{|l|}{ NHEM-PTEN $N^{\text {HemDel }}$} \\
\hline Pathways in cancer & 88 & 1.97E-06 \\
\hline PI3K-Akt signaling pathway & 66 & 4.05E-03 \\
\hline HTLV-I infection & 57 & $1.95 \mathrm{E}-04$ \\
\hline Rap1 signaling pathway & 55 & 2.00E-06 \\
\hline Lysosome & 51 & 4.36E-14 \\
\hline Focal adhesion & 49 & $1.14 \mathrm{E}-04$ \\
\hline Neuroactive ligand-receptor interaction & 49 & $4.92 \mathrm{E}-02$ \\
\hline Cytokine-cytokine receptor interaction & 48 & $2.79 \mathrm{E}-03$ \\
\hline \multicolumn{3}{|l|}{ NHEM-PTEN ${ }^{\text {HomDel }}$} \\
\hline Pathways in cancer & 54 & 4.46E-02 \\
\hline PI3K-Akt signaling pathway & 48 & $4.88 \mathrm{E}-02$ \\
\hline Rap1 signaling pathway & 39 & 7.93E-04 \\
\hline Focal adhesion & 34 & $1.18 \mathrm{E}-02$ \\
\hline Axon guidance & 31 & 2.12E-05 \\
\hline Lysosome & 26 & $9.01 \mathrm{E}-04$ \\
\hline Cell cycle & 24 & $5.99 \mathrm{E}-03$ \\
\hline Homologous recombination & 8 & $3.03 \mathrm{E}-02$ \\
\hline Other types of O-glycan biosynthesis & 8 & 4.24E-02 \\
\hline
\end{tabular}

diffgenes Jun

NHEM-PTEN ${ }^{W T}$ Jun

Pathways in cancer

$45 \quad 3.59 \mathrm{E}-05$

PI3K-Akt signaling pathway

Rap1 signaling pathway

$35 \quad 2.76 \mathrm{E}-03$

Focal adhesion

$32 \quad 2.39 \mathrm{E}-06$

$31 \quad 4.63 \mathrm{E}-06$
Table 1 continued

\begin{tabular}{lll} 
diffgenes Jun & & \\
\hline Proteoglycans in cancer & 25 & $8.45 \mathrm{E}-04$ \\
MAPK signaling pathway & 25 & $1.86 \mathrm{E}-02$ \\
Endocytosis & 25 & $2.14 \mathrm{E}-02$ \\
Ras signaling pathway & 24 & $8.73 \mathrm{E}-03$ \\
NHEM-PTEN ${ }^{\text {HemDel_Jun }}$ & & \\
Pathways in cancer & 31 & $5.11 \mathrm{E}-04$ \\
PI3K-Akt signaling pathway & 24 & $1.19 \mathrm{E}-02$ \\
Rap1 signaling pathway & 20 & $7.85 \mathrm{E}-04$ \\
Cytokine-cytokine receptor interaction & 20 & $2.30 \mathrm{E}-03$ \\
Focal adhesion & 19 & $1.60 \mathrm{E}-03$ \\
Ras signaling pathway & 18 & $9.70 \mathrm{E}-03$ \\
CAMP signaling pathway & 17 & $6.17 \mathrm{E}-03$ \\
Hippo signaling pathway & 15 & $3.02 \mathrm{E}-03$ \\
\hline
\end{tabular}

several differentially expressed PI3K/AKT-related genes in $P T E N^{\text {HomDel }}$ cells, which are regulated by $c$-Jun in $P T E N^{+}$cells. Figure 4a shows the overlap of the identified differentially expressed PI3K/AKT-related genes (RNASeq) and the $c$-Jun-regulated genes detected by ChIP-Seq in both subgroups, PTEN ${ }^{\mathrm{WT}}$ (9.94\% diff. exp.; 5.11\% c-Jun regulated) and PTEN ${ }^{\text {HemDel }}$ (7.95\% differentially expressed; $2.84 \% \quad c-J u n$ regulated). Figure $4 \mathrm{~b}$ displays the expression status of four differentially expressed PI3K/ AKT-related genes (PDGFB and CCND1 regulated by $c$ Jun in PTEN ${ }^{\mathrm{WT}}, C D K 6$, and BCL2 regulated by $c$-Jun in $P T E N^{\mathrm{HemDel}}$ ), the associated $c-J u n$ peaks (red arrows; ChIP-Seq) and the H3K27 acetylation status around the $c$ Jun peaks (blue), illustrating the differences in transcriptionally active genomic regions between NHEMs and melanoma cell lines. Moreover, we confirmed our findings by quantitative mRNA expression analysis of the identified differentially expressed c-Jun target genes (FGF5, CCND1, EGFR, PDGFB, LAMB3) after c-Jun siRNA transfection (Supplementary Fig. 3B). Our data clearly reveal the potential of the transcription factor $c$-Jun to either upregulated or downregulate target gene expression. All identified differentially expressed genes and those regulated by $c$-Jun of the analyzed melanoma subsets are presented in Supplementary Table 4. The previously described functions of each identified c-Jun regulated differentially expressed gene in melanoma are depicted in Supplementary Table 5.

Validation of c-Jun-dependent anti-apoptotic and prosurvival effects in early melanoma cells

To validate our findings, we performed knockdown experiments. First, we determined the total protein 
A PTEN ${ }^{W T}$

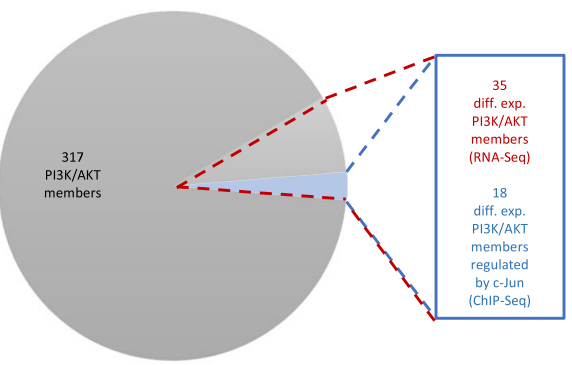

B

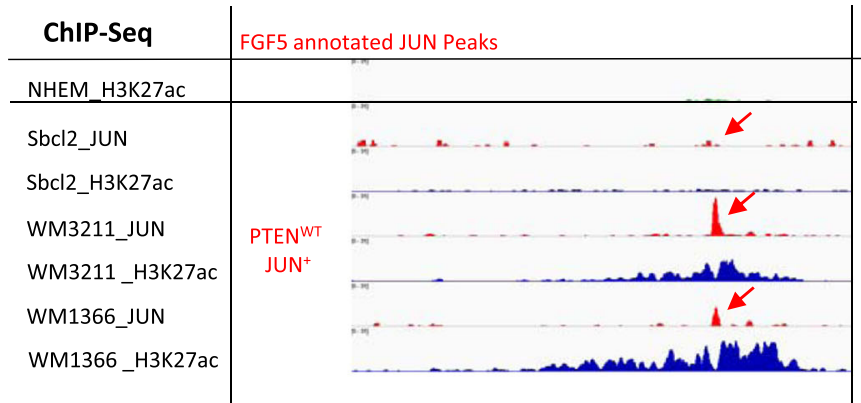

PTEN HemDel

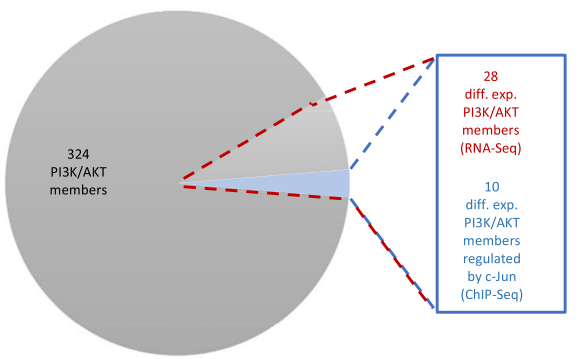

RNA-Seq

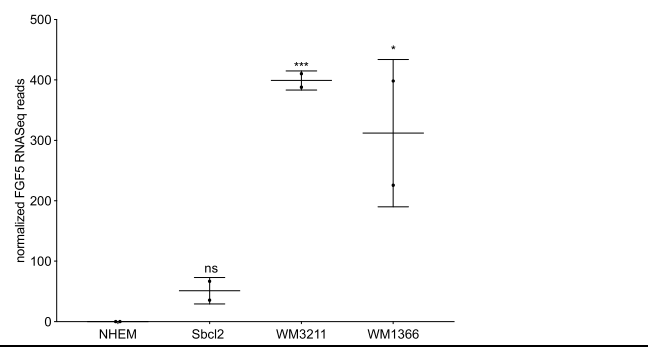

RNA-Seq

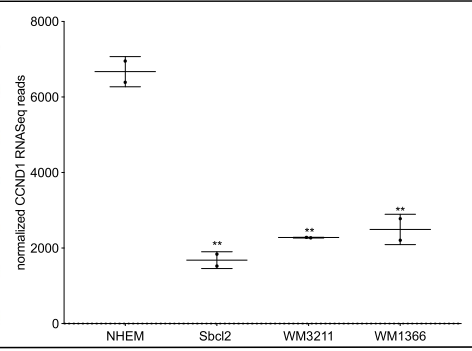

RNA-Seq

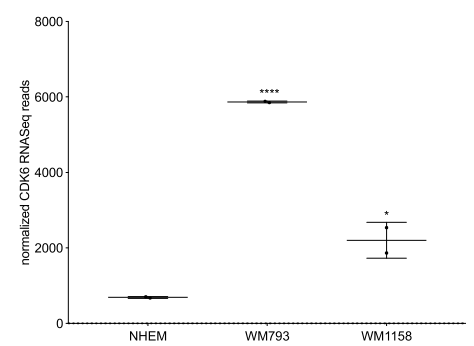

RNA-Seq

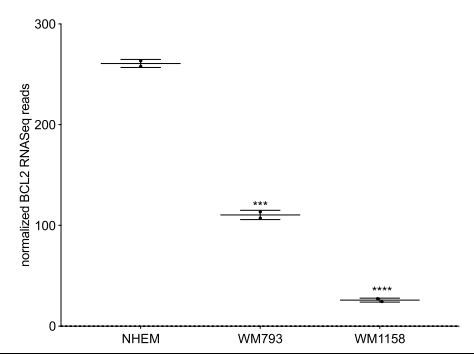

Figure 4

Fig. 4 (See legend on next page.) 
(see figure on previous page)

Fig. 4 C-Jun directly regulates PI3K/AKT members in PTEN-positive melanoma cells. a Differentially expressed PI3K/AKT members (PTEN ${ }^{\text {WT }}$ : 35/ 352; PTEN ${ }^{\text {HemDel: }}$ :28/352; RNA-Seq) overlap with c-Jun-regulated ones (PTEN ${ }^{W T}:$ 18/35; PTEN ${ }^{\text {HemDel: }}$ 10/28; ChIP-Seq). b Expression status (RNASeq) of four differentially expressed PI3K/AKT-related genes (PDGFB and CCND1 regulated by c-Jun in PTEN ${ }^{\mathrm{WT}}$, CDK6 and BCL2 regulated by c-Jun in PTEN ${ }^{\text {HemDel }}$ ), the associated c-Jun peaks (red arrows; ChIP-Seq) and the H3K27 acetylation status around the c-Jun peaks (blue), illustrating the differences in transcriptionally active genomic regions between NHEMs and melanoma cell lines. The box-plots show the mean \pm s.d. of read counts of two independent experiments $\left({ }^{* * * *} P<0.0001 ;{ }^{* * *} P<0.001 ;{ }^{* *} P<0.01 ;{ }^{*} P<0.5\right)$

amount of PTEN, c-Jun, and phospho-AKT in $P T E N^{+} / c$ -

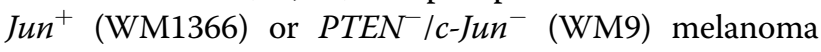
cells by Western blot analysis (Fig. 5a, b). We observed increased phospho- $A K T$ (P-AKT) in WM9 melanoma cells lacking PTEN and $c$-Jun expression. Thus, we hypothesized that $c$-Jun expression and transcriptional activity promotes survival in $P T E N$-expressing melanoma cells. Interestingly, $A K T$-siPool transfection in PTEN-/cJUn WM9 melanoma cells to mimic PTEN re-expression increased c-Jun protein expression (Fig. 5c, d). Moreover, we could validate our prior findings after $c$-Jun-siPool transfection in WM1366. Western blot analysis depicted a decreased $A K T$ activity (P-AKT) (Fig. 5e, f) and inhibition of $c$-Jun after siRNA transfection resulted in a significant decrease in cell number compared to sictrl-transfected cells (Fig. 5g). Transfection efficiencies are presented in Supplementary Fig. 6.

Importantly, these findings could also be validated by analyzing TCGA-sequencing data (https://www.cancer. gov/tcga) of a cohort of 471 melanoma patients (TCGA, Project ID: TCGA-SKCM). These data were categorized by the PTEN copy number alteration $\left(P T E N^{\mathrm{WT}}\right.$, PTENHemDel, $P T E N H$ HomDel $)$ and further analyzed regarding to the c-Jun expression status by using the cBioPortal ${ }^{18,19}$. The data reveal a significant downregulation of c-Jun in patients with PTEN ${ }^{\mathrm{HomDel}}$ and an almost unchanged $c$-Jun expression pattern in those with PTEN ${ }^{\mathrm{HemDel}}$ compared to $P T E N^{\mathrm{WT}}$ patients, supporting our findings (Fig. 5g).

\section{High c-Jun expression levels and loss of PTEN are linked to poor prognosis in melanoma patients}

Further analysis of the mRNA expression (RNA-Seq) showed a significant correlation of the expression of $c$-Jun and of PI3K/AKT-signaling members in skin cutaneous melanoma patients (TCGA, Project ID: TCGA-SKCM) confirming our findings (Fig. 6a). Further, we investigated overall survival of 471 skin cutaneous melanoma patients based on the PTEN and c-JUn expression status, and could clearly show that melanoma patients with alterations in both, c-JUn and PTEN, belong to the high risk group, whereas patients with either PTEN ${ }^{\mathrm{WT}}$ or $c$-Jun ${ }^{\mathrm{WT}}$ belong to the low risk group (Fig. 6b, c).

In summary, we identified a new, functional relevant relationship between the oncogenic transcription factor $c$ JUn and the tumor suppressor PTEN in malignant melanoma and a mechanism explaining the reduction in apoptosis and induction of tumor cell growth in PTEN ${ }^{+}$ melanoma cells, thus enabling the development of a malignant phenotype.

\section{Discussion}

In previous studies, we demonstrated that the transcription factor c-Jun plays a critical role in the development and progression of malignant melanoma ${ }^{3,5,9,10}$. However, the detailed molecular mechanism of c-Jun's influence on melanoma progression and development remains elusive given that only a subset of target genes is known. Previous studies have demonstrated that cancerrelevant genes, such as cyclin $D 1, p 53$, and $I N K 4 A^{20,21}$, are regulated by c-Jun. Moreover, we demonstrate that tumor-relevant genes, such as FosB, WEE1, PVR, $M A P 1 L C 3 B$, and $L G A L S 3$, are deregulated in melanoma by direct regulation via $c-J u n^{5}$.

In this study, we investigated the detailed molecular role of the transcription factor $c$-Jun in melanoma cell lines representing different tumor stages and mutation status as determined by RNA-Seq and ChIP-Seq experiments. Differential gene expression in melanoma compared to NHEMs differs according to melanoma stage and mutation status of the sequenced melanoma cell lines, indicating various possible mechanisms leading to a malignant phenotype. Consistent with our findings, Haqq and colleagues ${ }^{22}$ also discovered gene expression differences between radial growth phase (RGP) and vertical growth phase (VGP) melanoma cells. It is well established that critical tumorrelevant signaling pathways, such as MAPK and PI3K, are deregulated in melanoma cells, leading to a high proliferative, migratory, and invasive potential of these cells and overcoming apoptosis $^{23,24}$. Although oncogenic key players, such as BRAF, NRAS, $p 53$, and PTEN, within these pathways are well characterized, the detailed molecular mechanisms remain elusive.

Our analysis revealed that $c$-Jun expression status correlates with PTEN expression in melanoma cells, indicating a dependency of PTEN and c-Jun to allow the development of a malignant phenotype. Computational clustering of our sequencing data suggested a dependency of $c$-Jun activity in melanoma cells based on the presence of the tumor suppressor PTEN. Moreover, the number of c-Jun peaks decreased as PTEN expression decreased and was ultimately lost. Mutations in members of the PI3K pathway have been extensively studied in many cancer entities. In melanoma, 

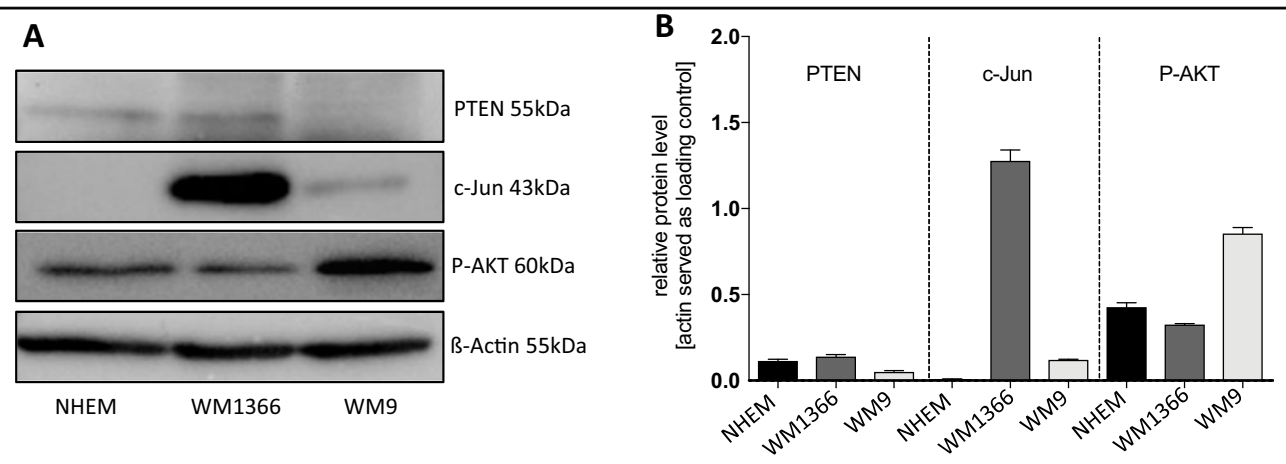

C
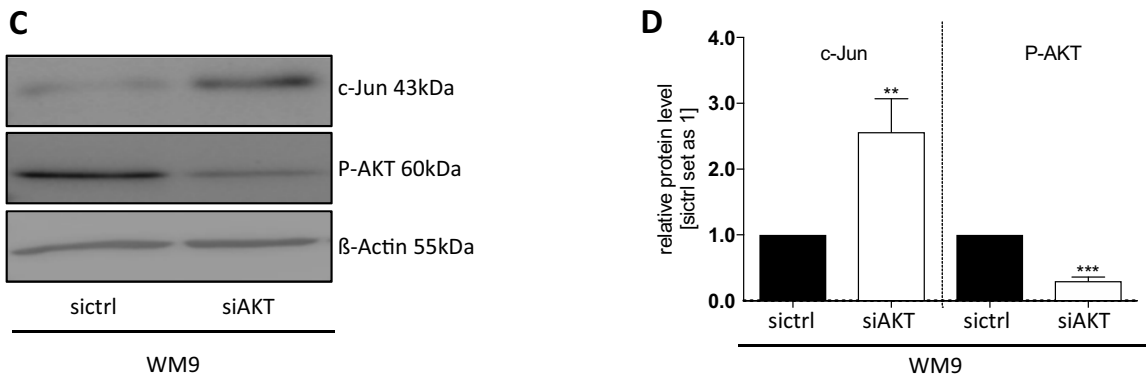

$\mathbf{E}$
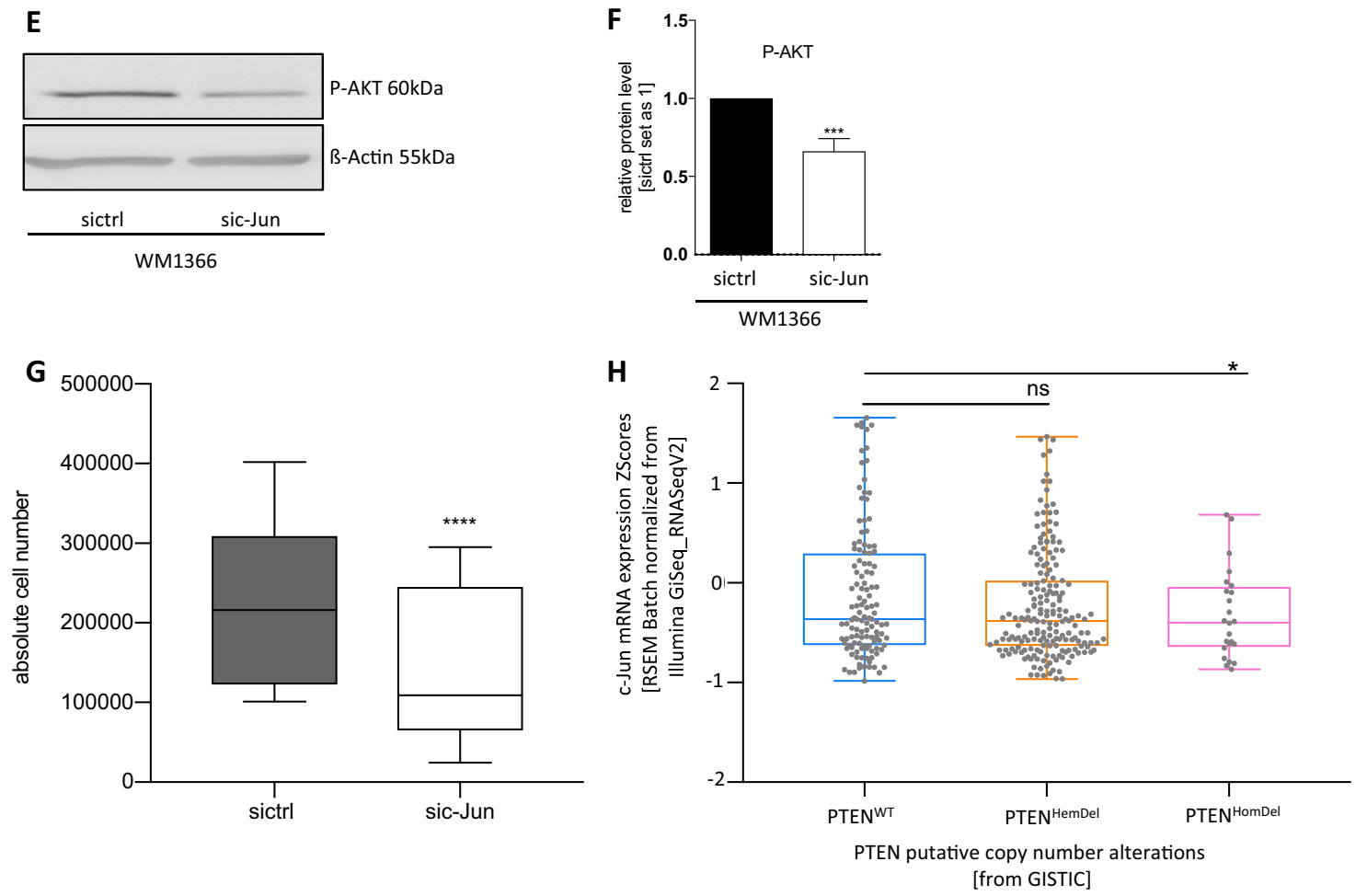

Fig. 5 c-Jun-dependent anti-apoptotic and pro-survival behavior of melanoma cells. a Western blot analysis revealing PTEN, C-JUn, and P-AKT protein levels in WM1366 and WM9 melanoma cell lines compared with NHEMs. ß-actin served as loading control. b Densitometry of the PTEN, c-Jun, and P-AKT protein level compared to B-actin. c Western blot analysis after AKT-siPool transfection of WM9 melanoma cells revealed increased c-Jun protein expression compared to sictrl-transfected cells. B-actin served as loading control. d Densitometry of the $c$-Jun and P-AKT protein level after AKT knockdown compared to ß-actin. e Western blot analysis after c-Jun-siPool transfection show an decreased AKT activity (P-AKT). B-actin served as loading control. $\mathbf{f}$ Densitometry of the P-AKT protein level after $c$-Jun knockdown compared to B-actin. $\mathbf{g}$ Total cell amount after $c$-Jun knockdown by siRNA transfection compared to the amount of sictrl-transfected cells. A significant decrease of cell survival in the sic-Jun knockdown samples is depicted. $\mathbf{h}$ Analysis of TGCA sequencing data of a cohort of 471 melanoma patients. Data were categorized by the PTEN copy number alteration (PTEN ${ }^{W T}$, PTEN ${ }^{\text {HemDel }}$, PTEN $\left.N^{\text {HomDel }}\right)$ and further analyzed regarding to the $c$-Jun expression status by cBioPortal $\left({ }^{*} P<0.05\right.$, Welch's test, one-sided). The box-plots show the mean \pm s.d. of three independent experiments; measurements were performed in triplicate $\left({ }^{* * * *} P<0.0001\right.$; ${ }^{* * *} P<0.001$; ${ }^{* *} P<$ $0.01)$ 

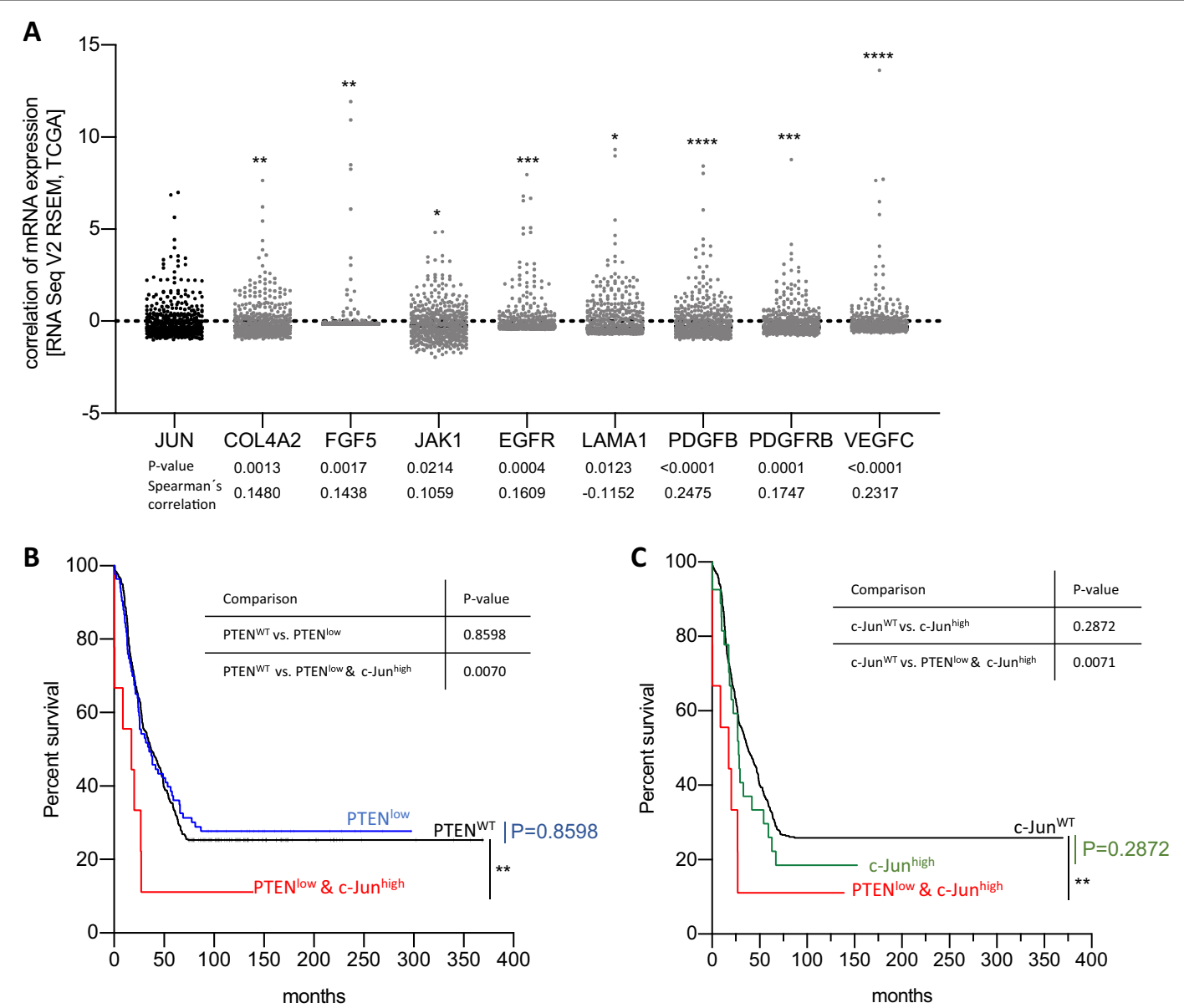

Fig. 6 High c-Jun expression levels and loss of PTEN are linked to poor prognosis in melanoma patients. a mRNA expression (RNA-Seq) of CJun and PI3KJAKT-signaling members show a significant correlation in skin cutaneous melanoma patients (TCGA, Project ID: TCGA-SKCM). Spearman's correlation is shown for each comparison. The scatter plots show the $\log 2(x+1)$ transformed RSEM normalized read counts of each melanoma patient $\left({ }^{* * *} P<0.0001 ;{ }^{* * *} P<0.001 ;{ }^{* *} P<0.01 ;{ }^{*} P<0.5\right)$. b, c Survival rates of the 471 skin cutaneous melanoma patients (TCGA, Project ID: TCGASKCM) depicted by PTEN and/or c-Jun expression. Comparison of the survival curves is illustrated in tables (**P $<0.001$, Log-rank (Mantel-Cox) test)

PTEN inactivation could be identified as the crucial step activating the PI3K pathway ${ }^{25-27}$. Consistently, recent studies also demonstrate a correlation between $c$-Jun and PTEN in nonmelanoma cancer. Hettinger and colleagues identified a direct downregulation of PTEN by $c$-Jun, leading to an upregulation of the $A K T$ survival pathway in several cancer cells, such as pancreatic cells, glioblastoma cells, and transformed kidney epithelial cells ${ }^{28}$. Moreover, these results could also be confirmed in lung cancer cells ${ }^{13}$. However, the described PTEN and c-Jun expression pattern and binding site of $c$-Jun by Hettinger et al. was not revealed in our data, suggesting a different molecular relation between PTEN and c-Jun in melanoma.

Our data clearly revealed a functional correlation of $c$ JUn and PTEN in melanoma cells. In accordance with this finding, recent studies identified a crosstalk mechanism, whereby constitutive ERK activity suppresses PTEN in melanoma models and is mediated by modulation of $c$ -
$J_{U n}{ }^{29}$. Furthermore, crosstalk between JNK and PTEN and thus the PI3K/AKT pathway could be detected in invasive adenocarcinoma of the prostate ${ }^{30}$. Interestingly, a mechanism exists that links ERK to JNK signaling in human melanoma. Hyperactivated ERK increases the stability of $c$-Jun protein and induces $c$-Jun transcription by CREB activation. C-Jun subsequently induces RACK1 transcription, which enhances JNK activation. Consequently, $c-J u n$ is further stabilized and activated by JNK ${ }^{31}$.

Taken together, both identified crosstalk mechanisms support the hypothesis that c-Jun upregulation is the main link between the ERK/JNK-signaling pathways and the PI3K pathway, to overcome the tumor suppressive effect of PTEN, which would otherwise counteract the transformation process by inducing apoptosis.

Functional annotation analyses via multiple tools confirmed our findings. Analyses of the identified differentially expressed genes of melanoma cells resulted in a 
PTEN-independent enrichment of PI3K/AKT-signaling targets. Interestingly, we detected no increased $A K T$ activity in PTEN-expressing melanoma cells and thus investigated if $c$-Jun regulates PI3K/AKT-related genes to promote malignancy. Indeed, our data revealed direct regulation of PI3K/AKT targets via c-Jun but not $A K T$ itself, suggesting an overruling effect of $c$-Jun in the presence of PTEN during early melanoma development.

Previous studies described that increased levels of $c$-Jun in melanoma cell lines coincide with upregulation of phosphoinositide-dependent kinase 1 (PDK1) and phosphorylation of protein kinase $\mathrm{C}(\mathrm{PKC})$ and $A K T^{32}$. Although, prior studies reported that $c$-Jun is a negative regulator inhibiting PTEN, detailed mechanisms by which c-Jun inhibits PTEN and thus indirectly increases $A K T$ activity remain to be established ${ }^{33,34}$. Our data clearly showed no detectable alterations in $A K T$ expression and activity in $c$ JUn and PTEN-coexpressing melanoma cells. Thus, c-Jun seems to play a crucial role in the presence of PTEN to enable the development of a malignant phenotype, which could be confirmed by various knockdown experiments. Previous data from Chen and colleagues support our results, revealing that PTEN inactivation results in cellular senescence in mouse embryonic fibroblasts ${ }^{35}$. Given that melanomas preferentially activate the PI3K pathway through inactivation of PTEN, it can be hypothesized that c-Jun expression in early melanoma stages protects melanoma cells with decreasing PTEN levels from this growth arrest.

Taken together, our study confirms a crucial role of the transcription factor $c$-Jun in melanoma development and progression in $P T E N^{+}$melanoma cells to overcome apoptosis and promote malignancy not only in vitro but also in vivo. TCGA data analysis by cBioPortal confirm and support our latest findings. Thus, the detection of $c$ JUn and PTEN coexpression in melanoma represents a promising diagnostic marker for highly aggressive melanoma cells with the potential to result in MET with the loss of both c-Jun and PTEN.

Further investigations will be necessary to determine the detailed tumor-promoting consequences of c-Jun activity in malignant melanoma.

\section{Material and methods \\ Cell culture}

Human melanoma cell lines Sbcl-2, WM3211, WM1366, WM793, WM1158, and WM9 (a generous gift from Dr. M. Herlyn, Wistar Institute, Philadelphia, USA) derived from RGP (Sbcl-2), VGP (WM3211, WM1366, WM793), and melanoma MET (WM1158, WM9) were maintained in a culture medium consisting of MCDB153 (Sigma-Aldrich, Steinheim, Germany) with 20\% Leibovitz's L-15 (PAA Laboratories, Coelbe, Germany), 2\% FCS, $1.68 \mathrm{mM} \mathrm{CaCl} 2$ (Sigma), and $5 \mu \mathrm{g} / \mathrm{ml}$ insulin (SigmaAldrich, Steinheim, Germany) at $37^{\circ} \mathrm{C}$ and $5 \% \mathrm{CO}_{2}$.
NHEM were derived from neonatal foreskin (NHEMs, PromoCell, Heidelberg, Germany) and were cultured in melanocyte growth media $\mathrm{M} 2$ at $37^{\circ} \mathrm{C}$ and $5 \% \mathrm{CO}_{2}$.

\section{Western blotting}

Western blot analysis was performed as described previously ${ }^{5}$ using one of the following antibodies: anti-c-Jun (1 in 1000 dilution; Cell Signaling, Frankfurt am Main, Germany), anti-GAPDH ( 1 in 1000 dilution; Cell Signaling), anti $B$-Actin ( 1 in 3000 dilution; Sigma Aldrich), anti-PTEN (1 in 40 dilution; Santa Cruz), anti-AKT (1 in 2000 dilution; Cell Signaling, Frankfurt am Main, Germany), and anti-P-AKT (1 in 2000 dilution; Cell Signaling, Frankfurt am Main, Germany). After three washes with TBS-T, the membrane was incubated for $1 \mathrm{~h}$ with an alkaline phosphate-coupled secondary anti-mouse (1 in 3000 dilution in TBS-T) or anti-rabbit (1 in 3000 dilution in TBS-T) IgG antibody (Chemicon, Hofheim, Germany), respectively HRP-coupled antibodies and then washed thrice in TBS-T. Finally, immunoreactions were visualized by NBT/BCIP (Sigma-Aldrich) staining or Clarity TM Western ECL Substrate (Biorad).

\section{siRNA transfection experiments with siRNA-Pools}

siRNA transfection of WM1366 $\left(P T E N^{+} / c-J u n^{+}\right)$and WM9 $\left(P T E N^{-} / c-J u n^{+}\right)$melanoma cells was performed using the reverse transfection protocol of the Lipofectamine RNAiMAX reagent (Invitrogen, Carlsbad, CA, USA) according to the manufacturer's instructions using siRNAPools as described previously ${ }^{36}$. For transfection experiments, $2 \times 10^{5}$ cells were seeded each well in six-well plates. We applied "si-POOL-AKT", "si-POOL-PTEN", and "siPOOL-c-Jun" (functionally verified, by siTOOLs Biotech $\mathrm{GmbH}$, Planegg, Germany) for specific knockdown of $A K T 1, A K T 2, A K T 3, P T E N$, and $c$-Jun, respectively.

\section{Analysis of gene expression by quantitative PCR}

cDNAs of total RNA fractions were generated using Super-Script II Reverse Transcriptase Kit (Invitrogen, Groningen, The Netherlands). qRT-PCR was performed on a LightCycler (Roche, Mannheim, Germany) as described previously ${ }^{5}$. Annealing and melting temperatures were optimized for each primer set (Table 2). ßActin was used for normalization.

\section{RNA-Seq library preparation and mapping}

RNA-Seq samples and libraries were prepared as described previously ${ }^{37}$. Library preparation was performed with at least two biological replicates. Sequencing was performed according to the paired-end RNA sequencing protocols from Illumina on a HiSeq2000 with paired-end module (Illumina, Inc.). Fifty samples were sequenced from each side of a fragment $\sim 100 \mathrm{bp}$ long with an average number 20 million reads per sample. 


\begin{tabular}{|c|c|c|c|}
\hline Gene & Primer sequences (fwd/rev) & $T_{\mathrm{a}}\left({ }^{\circ} \mathrm{C}\right)$ & $T_{\mathrm{M}}\left({ }^{\circ} \mathrm{C}\right)$ \\
\hline B-actin & $\begin{array}{l}\text { 5'-CTACGTCGCCCTGGACTTCGAGC-3' } \\
\text { 5'-GATGGAGCCGCCGATCCACACGG-3' }\end{array}$ & $60-68$ & 80 \\
\hline c-Jun & $\begin{array}{l}\text { 5'-TTCCTCCCGTCCGAGAGCGG-3' } \\
5^{\prime} \text {-TCGGCGTGGTGGTGATGTGC-3' }\end{array}$ & $60-70$ & 80 \\
\hline AKT1 & $\begin{array}{l}\text { 5'-AGCCCACCCTTCAAGCCCCA-3' } \\
\text { 5'-CTGCGCTCGCTGTCCACACA-3' }\end{array}$ & 60 & 82 \\
\hline AKT3 & $\begin{array}{l}\text { 5'-TCTGCCTTGGACTATCTACA-3' } \\
\text { 5'-AATIIITATGTGGCCATCT-3' }\end{array}$ & 60 & 76 \\
\hline PTEN & $\begin{array}{l}\text { 5'-TGTGGTCTGCCAGCTAAAGG-3' } \\
\text { 5'-AGGTTCCTCTGGTCCTGGT-3' }\end{array}$ & 60 & 80 \\
\hline
\end{tabular}

Paired-end reads were aligned to the human reference genome sequence (hg38) using the STAR alignment software $(\mathrm{v} 2.5 .2 \mathrm{a})^{38}$. Only reads that mapped to a single unique location were considered for further analysis. Counts for RNA-Seq reads were calculated using the feature-counts software (v 1.4.6-p5).

\section{Chromatin immunoprecipitation}

ChIP was performed with the human melanoma cell lines Sbcl-2, WM3211, WM1366, WM793, WM1158, WM9 and NHEM that had not exceeded six passages. In terms of ChIP experiments with transcription factors, we performed double crosslinking with disuccinimidyl glutarate (DSG, Thermo Scientific, Rockford, USA) before a fixation step with formaldehyde. Chromatin from $10 \times 10^{6}$ cells of each cell line was crosslinked in 1/10th volume of fixation buffer (50 mM HEPES/KOH, pH 7.9, 11\% formaldehyde) for $10 \mathrm{~min}$ at room temperature and quenched by $0.125 \mathrm{M}$ glycine. After two washes with PBS and PMSF, the cells were scraped and centrifuged at $4{ }^{\circ} \mathrm{C}$ and $3500 \times g$ for $10 \mathrm{~min}$. The supernatant was discarded, and the pellet was resuspended in $15 \mathrm{ml}$ of lysis buffer 1 (5 mM PIPES [pH 8.0], $85 \mathrm{mM} \mathrm{KCl}$, 0.5\% NP-40, 1x Roche Complete, EDTA-free protease inhibitor) and incubated for $10 \mathrm{~min}$ on ice. After centrifugation of $5 \mathrm{~min}$ at $4{ }^{\circ} \mathrm{C}$ and $3500 \times g$, the supernatant was discarded, and the pellet resuspended in $15 \mathrm{ml}$ lysis buffer 2 (50 mM Tris-HCl, pH 8.0, 10 mM EDTA, 1\% SDS, $1 \times$ Roche Complete, EDTA-free protease inhibitor). The suspension was then incubated on ice for an additional $10 \mathrm{~min}$ and was examined under the microscope for quality assessment of nuclear prep. The nuclei were pelleted at $4{ }^{\circ} \mathrm{C}$ and $3500 \times g$ for $5 \mathrm{~min}$. The supernatant was discarded, and the pellet was resuspended in the desired volume of sonication buffer $\left(10 \times 10^{6}\right.$ cells in $450 \mu \mathrm{l}$ sonication buffer: $16.7 \mathrm{mM}$ Tris- $\mathrm{HCl}$ [pH 8.0], $1.2 \mathrm{mM}$ EDTA, $167 \mathrm{mM} \mathrm{NaCl}$, $0.01 \%$ SDS, $1.1 \%$ Triton X-100, $1 \times$ Roche Complete, EDTAfree protease inhibitor). Cross-linked chromatin was sheared to an average DNA fragment size of $~ 400-600$ bp using a Branson Sonifier 250 (Danbury, CT). After centrifugation,
$2 \%$ of supernatant was used as input. After preclearing with Sepharose CL-4B beads (Sigma) for $2 \mathrm{~h}$, chromatin samples from $10 \times 10^{6}$ cells were immunoprecipitated overnight with $5 \mu \mathrm{g}$ of rabbit polyclonal antibody anti-c-Jun (Santa Cruz Biotechnology Inc.) or anti- H3K37ac (Abcam). Immunocomplexes were recovered by $3 \mathrm{~h}$ of incubation with protein A-Sepharose beads (GE Healthcare) at $4{ }^{\circ} \mathrm{C}$. These beads were washed thrice and resuspended in $1 \mathrm{mM}$ EDTA and $10 \mathrm{mM}$ Tris- $\mathrm{HCl}, \mathrm{pH}$ 8.1. Precipitates were serially washed with $400 \mu \mathrm{l}$ Washing Buffer I $(2 \mathrm{mM}$ EDTA, $20 \mathrm{mM}$ Tris-HCl [pH 8.0], 0.1\% SDS, $1 \%$ Triton X-100, $150 \mathrm{mM}$ $\mathrm{NaCl})$, Washing Buffer III (1 mM EDTA, $10 \mathrm{mM}$ Tris- $\mathrm{HCl}$ [pH 8.0], 1\% NP-40, 1\% Deoxycholate, $0.25 \mathrm{M} \mathrm{LiCl})$ and thrice with $1 \mathrm{mM}$ EDTA and $10 \mathrm{mM}$ Tris- $\mathrm{HCl}, \mathrm{pH} 8.0$. Precipitated chromatin complexes were removed from the beads through a 20-min incubation with $100 \mu$ of $1 \%$ SDS, $0.1 \mathrm{M} \mathrm{NaHCO}$ with vortexing each $5 \mathrm{~min}$. This step was repeated twice with 10 -min incubation times. After reverse cross-linking, DNA was purified using the QIAquick PCR purification kit (Qiagen).

\section{ChIP-seq and mapping}

DNA from ChIP (10-50 ng) was adapter ligated and polymerase chain reaction amplified according to the manufacturer's instructions (Illumina, San Diego, USA). ChIP fragments were sequenced for 36 cycles on Illumina Genome Analyzer according to the manufacturer's protocol. Sequence tags of all experiments were mapped to the current human reference sequence (GRCh8/hg38) using Bowtie 2 (v 2.2.7) ${ }^{39}$, and only uniquely mapped tags were used for downstream analysis. Tag counts were normalized to $10^{7}$ specifically mapped tags.

\section{Sequencing data analysis}

The raw RNA-Seq counts were used for differential gene expression analysis that was performed using DESeq2 ( $v$ 1.14.1 $)^{40}$. Differentially expressed genes with a false-discovery rate (FDR) $<0.05$ were regarded as statistically significant. Normalization was performed by library size based on the raw counts.

Genome Ontology annotation and RNA-Seq reads annotation were performed using scripts provided by STAR (based on GENCODE V24).

Analysis of mapped ChIP-seq tags was performed using HOMER, which is freely available at http://biowhat.ucsd. edu/homer $/{ }^{41}$. The ChIP-Seq sequence data were assessed for abnormal GC content, excessive clonal amplification (multiple tags starting at the same genomic position), inherent sequence bias in the sequenced tags and surrounding genomic positions and contamination with plasmid/cDNA sequences. The identification of ChIP-Seq peaks (bound regions) was performed using a custom approach (HOMER) that combines features of previously published methods. Peaks were defined at a 0.001 
estimated false discovery rate. Genome Ontology annotation and ChIP-Seq tag annotation of peak sets was performed using scripts provided by HOMER (based on GENCODE V24). Next generation sequencing data used in this study are listed below:

\begin{tabular}{llll}
\hline ChIP-seq & & & \\
\hline Cell type & $\begin{array}{l}\text { Target } \\
\text { protein }\end{array}$ & $\begin{array}{l}\text { Total uniquely mapped } \\
\text { tags (hg38) }\end{array}$ & $\begin{array}{l}\text { Total peaks } \\
\text { identified }\end{array}$ \\
\hline NHEM & H3K27ac & $22,173,094$ & 72,031 \\
Sbcl-2 & c-Jun & $8,977,157$ & 54,943 \\
Sbcl-2 & H3K27ac & $16,073,130$ & 66,130 \\
WM3211 & c-Jun & $14,381,672$ & 91,277 \\
WM3211 & H3K27ac & $29,299,544$ & 87,743 \\
WM1366 & c-Jun & $14,015,631$ & 28,061 \\
WM1366 & H3K27ac & $16,732,104$ & 71,798 \\
WM793 & c-Jun & $12,148,216$ & 29,726 \\
WM793 & H3K27ac & $21,459,505$ & 79,942 \\
WM1158 & c-Jun & $15,632,274$ & 28,000 \\
WM1158 & H3K27ac & $17,825,517$ & 78,432 \\
WM9 & c-Jun & $13,634,991$ & 1164 \\
WM9 & H3K27ac & $18,466,555$ & 73,667 \\
\hline
\end{tabular}

RNA-Seq

\begin{tabular}{ll} 
Cell type & Total mapped read counts (hg38) \\
\hline NHEM_rep1 & $10,306,403$ \\
NHEM_rep2 & $15,287,883$ \\
Sbcl-2_rep1 & $9,710,494$ \\
Sbcl-2_rep2 & $9,217,901$ \\
WM3211_rep1 & $6,644,449$ \\
WM3211_rep2 & $8,660,603$ \\
WM1366_rep1 & $5,447,622$ \\
WM1366_rep2 & $11,600,401$ \\
WM793_rep1 & $9,590,158$ \\
WM793_rep2 & $9,065,432$ \\
WM1158_rep1 & $12,014,897$ \\
WM1158_rep2 & $6,173,661$ \\
WM9_rep1 & $15,814,898$ \\
WM9_rep2 & $3,534,649$ \\
\hline
\end{tabular}

\section{Attachment assay using the xCELLigence system}

Attachment was determined using the xCELLigence System of Roche Diagnostics (Penzberg, Germany) as described previously ${ }^{42}$. This instrument monitors the behavior of the cells in real time by measuring the electrical impedance across interdigitated microelectrodes covering the bottom of 'E-plates'. Electrode impedance is displayed as cell index values.

\section{Cell proliferation assay}

Melanoma cells (4000 cells/well) were seeded in a 96well plate, the medium was aspirated and cells were incubated with the dye-binding solution from the CyQUANT $^{\circ}$ NF Cell Proliferation Assay Kit (Invitrogen) according to the manufacturer's protocol. Finally, fluorescence was measured in an EMax Microplate Reader (MWG Biotech, Ebersberg, Germany) after 1 and $72 \mathrm{~h}$.

\section{Migration assay}

Migration potential of melanoma cells was quantified using the Cultrex 96-Well Cell Migration Assay (Trevigen, Gaithersburg, USA) according to the manufacturer's instructions as described previously ${ }^{43}$. Briefly, melanoma cells were seeded into the upper compartment of the provided 96-well plate (20,000 cells/well) in RPMI. The lower compartment was filled with RPMI supplemented with conditioned medium from fibroblasts and 20\% (directed migration) or $0 \%$ (undirected migration) FCS, respectively, as chemoattractants. After incubation at $37^{\circ} \mathrm{C}$ for $4 \mathrm{~h}$ cell migration was quantified by fluorimetry with an EMax Microplate Reader (MWG Biotech, Ebersberg, Germany).

\section{GO analysis}

The Database for Annotation, Visualization, and Integrated Discovery (DAVID; v 6.8) (4,,45 $^{4}$ was used to investigate biological meaning of the obtained differential expressed gene lists by DESeq2 of our RNA-Seq data and also GO terms for differentially expressed genes potentially regulated by $c$-Jun. The STRING database ( $\mathrm{v} 10.5)^{46,47}$ was used to visualize predicted associations of differentially expressed genes or differentially expressed genes potentially regulated by $c$-Jun, respectively. Moreover, enriched GO terms of different gene sets were also identified by STRING.

Gene Set enrichment analyses were performed using GSEA (v 2.0) $)^{48,49}$.

GREAT $^{50,51}$ was used to identify GO terms of noncoding genomic regions (ChIP-Seq peaks) by analyzing the annotations of the nearby genes. The association rule was set as follows: proximal, $10 \mathrm{~kb}$ upstream and $10 \mathrm{~kb}$ downstream (any gene in this interval relative to input regions is included); plus distal, up to $1000 \mathrm{~kb}$ (if no gene is present in the proximal interval, the closest gene in this distal interval is included).

\section{Statistical analysis}

In the bar graphs, results are expressed as mean \pm s.d. (range). Comparisons between qRT-PCR data were made 
using the Student's unpaired $t$-test. A $P$-value of $<0.05$ was considered as statistically significant (NS: not significant, ${ }^{*} P<0.05,{ }^{* * *} P<0.01, \quad{ }^{* * * *} P<0.001,{ }^{* * * * *} P<0.0001$ ). Group comparisons of Next Generation Sequencing data were made by the non-parametric Wilcoxon test and of TCGA patient data by the Welch's $t$-test after removing identified outliers. All calculations were performed using the GraphPad Prism Software (V8, GraphPad Software, Inc., San Diego, CA, USA) or RStudio (Version: 1.1.456, RStudio, Inc., Boston, MA, URL, http://www.rstudio.com).

\section{Acknowledgements}

We thank the biomedical sequencing facility (BSF) of the CeMM (Vienna; Austria), the KFB (Kompetenzzentrum Fluoreszente Bioanalytik; Regensburg; Germany) and the Genomics Platform of the Regensburg Center for Interventional Immunology (RCl, University Regensburg and University Medical Center Regensburg, Germany) for sequencing and help with NGS library preparation. This work was supported by a grant from the German Cancer Aid to A.K.B.

\section{Author details}

${ }^{1}$ Institute of Biochemistry (Emil-Fischer Center), Friedrich-Alexander University Erlangen-Nürnberg, Erlangen, Germany. ${ }^{2}$ Faculty of Applied Health Care Sciences, University of Applied Science, Deggendorf, Germany. ${ }^{3}$ Department of Internal Medicine III, University Hospital Regensburg, Regensburg, Germany. ${ }^{4}$ Regensburg Center for Interventional Immunology (RCI), University Regensburg and University Medical Center Regensburg, 93053 Regensburg, Germany. ${ }^{5}$ Comprehensive Cancer Center (CCC)-EMN, Erlangen, Germany

\section{Conflict of interest}

The authors declare that they have no conflict of interest.

\section{Publisher's note}

Springer Nature remains neutral with regard to jurisdictional claims in published maps and institutional affiliations.

Supplementary Information accompanies this paper at (https://doi.org/ 10.1038/s41419-019-1821-9).

Received: 1 April 2019 Revised: 29 June 2019 Accepted: 16 July 2019 Published online: 05 August 2019

\section{References}

1. Shaulian, E. AP-1-The Jun proteins: oncogenes or tumor suppressors in disguise? Cell Signal. 22, 894-899 (2010).

2. Shaulian, E. \& Karin, M. AP-1 in cell proliferation and survival. Oncogene 20, 2390-2400 (2001)

3. Kappelmann, M., Bosserhoff, A. \& Kuphal, S. AP-1/C-Jun transcription factors: regulation and function in malignant melanoma. Eur. J. Cell Biol. 93, 76-81 (2014).

4. Eferl, R. \& Wagner, E. F. AP-1: a double-edged sword in tumorigenesis. Nat. Rev. Cancer 3, 859-868 (2003).

5. Schummer, P., Kuphal, S., Vardimon, L., Bosserhoff, A. K. \& Kappelmann, M. Specific c-Jun target genes in malignant melanoma. Cancer Biol. Ther. 17, 486-497 (2016).

6. Lopez-Bergami, P., Lau, E. \& Ronai, Z. Emerging roles of ATF2 and the dynamic AP1 network in cancer. Nat. Rev. Cancer 10,65-76 (2010).

7. Jochum, W., Passegue, E. \& Wagner, E. F. AP-1 in mouse development and tumorigenesis. Oncogene 20, 2401-2412 (2001).

8. Weiss, C. \& Bohmann, D. Deregulated repression of c-Jun provides a potential link to its role in tumorigenesis. Cell Cycle 3, 111-113 (2004).

9. Kappelmann, M., Kuphal, S., Meister, G., Vardimon, L. \& Bosserhoff, A. K. MicroRNA miR-125b controls melanoma progression by direct regulation of cJun protein expression. Oncogene 32, 2984-2991 (2013).
10. Spangler, B. et al. ETS-1/RhoC signaling regulates the transcription factor c-Jun in melanoma. Int. J. Cancer 130, 2801-2811 (2012).

11. Spangler, B., Vardimon, L., Bosserhoff, A. K. \& Kuphal, S. Post-transcriptional regulation controlled by E-cadherin is important for c-Jun activity in melanoma. Pigment Cell Melanoma Res. 24, 148-164 (2011).

12. Parmiter, A. H., Balaban, G., Clark, W. H. Jr. \& Nowell, P. C. Possible involvement of the chromosome region 10q24-q26 in early stages of melanocytic neoplasia. Cancer Genet. Cytogenet. 30, 313-317 (1988).

13. Vasudevan, K. M., Burikhanov, R., Goswami, A. \& Rangnekar, V. M. Suppression of PTEN expression is essential for antiapoptosis and cellular transformation by oncogenic Ras. Cancer Res. 67, 10343-10350 (2007).

14. Agarwal, S. K. et al. Menin interacts with the AP1 transcription factor JunD and represses JunD-activated transcription. Cell 96, 143-152 (1999).

15. Huang da, W., Sherman, B. T. \& Lempicki, R. A. Systematic and integrative analysis of large gene lists using DAVID bioinformatics resources. Nat. Protoc. 4, 44-57 (2009).

16. Huang da, W., Sherman, B. T. \& Lempicki, R. A. Bioinformatics enrichment tools: paths toward the comprehensive functional analysis of large gene lists. Nucleic Acids Res. 37, 1-13 (2009).

17. Thorvaldsdottir, H., Robinson, J. T. \& Mesirov, J. P. Integrative Genomics Viewer (IGV): high-performance genomics data visualization and exploration. Brief Bioinform. 14, 178-192 (2013).

18. Cerami, E. et al. The cBio cancer genomics portal: an open platform for exploring multidimensional cancer genomics data. Cancer Discov. 2, 401-404 (2012).

19. Gao, J. et al. Integrative analysis of complex cancer genomics and clinical profiles using the cBioPortal. Sci. Signal. 6, pl1 (2013).

20. Bakiri, L., Lallemand, D., Bossy-Wetzel, E. \& Yaniv, M. Cell cycle-dependent variations in C-Jun and JunB phosphorylation: a role in the control of cyclin D1 expression. EMBO J. 19, 2056-2068 (2000)

21. Schreiber, M. et al. Control of cell cycle progression by c-Jun is p53 dependent Genes Dev. 13, 607-619 (1999).

22. Haqq, C. et al. The gene expression signatures of melanoma progression. Proc. Natl Acad. Sci. USA 102, 6092-6097 (2005).

23. Fecher, L. A., Amaravadi, R. K. \& Flaherty, K. T. The MAPK pathway in melanoma. Curr. Opin. Oncol. 20, 183-189 (2008).

24. Davies, M. A. The role of the PI3K-AKT pathway in melanoma. Cancer J. 18, 142-147 (2012).

25. Madhunapantula, S. V. \& Robertson, G. P. The PTEN-AKT3 signaling cascade as a therapeutic target in melanoma. Pigment Cell Melanoma Res. 22, 400-419 (2009).

26. Milella, M. et al. PTEN: multiple functions in human malignant tumors. Front. Oncol. 5, 24 (2015).

27. Stahl, J. M. et al. Loss of PTEN promotes tumor development in malignant melanoma. Cancer Res. 63, 2881-2890 (2003).

28. Hettinger, K. et al. c-Jun promotes cellular survival by suppression of PTEN. Cell Death Differ. 14, 218-229 (2007).

29. Ciuffreda, L. et al. The mitogen-activated protein kinase (MAPK) cascade controls phosphatase and tensin homolog (PTEN) expression through multiple mechanisms. J. Mol. Med. 90, 667-679 (2012).

30. Hubner, A. et al. JNK and PTEN cooperatively control the development of invasive adenocarcinoma of the prostate. Proc. Natl Acad. Sci. USA 109 12046-12051 (2012)

31. Lopez-Bergami, P. et al. Rewired ERK-JNK signaling pathways in melanoma Cancer Cell 11, 447-460 (2007).

32. Lopez-Bergami, P. et al. c-Jun regulates phosphoinositide-dependent kinase 1 transcription: implication for Akt and protein kinase $C$ activities and melanoma tumorigenesis. J. Biol. Chem. 285, 903-913 (2010).

33. Gericke, A., Munson, M. \& Ross, A. H. Regulation of the PTEN phosphatase. Gene 374, 1-9 (2006).

34. Song, M. S., Salmena, L. \& Pandolfi, P. P. The functions and regulation of the PTEN tumour suppressor. Nat. Rev. Mol. Cell Biol. 13, 283-296 (2012).

35. Chen, J. et al. MicroRNA-193b represses cell proliferation and regulates cyclin D1 in melanoma. Am. J. Pathol. 176, 2520-2529 (2010).

36. Dietrich, P., Kuphal, S., Spruss, T., Hellerbrand, C. \& Bosserhoff, A. K. Wild-type KRAS is a novel therapeutic target for melanoma contributing to primary and acquired resistance to BRAF inhibition. Oncogene 37, 897-911 (2018).

37. Andersson, R. et al. An atlas of active enhancers across human cell types and tissues. Nature 507, 455-461 (2014).

38. Dobin, A. \& Gingeras, T. R. Optimizing RNA-Seq mapping with STAR. Methods Mol. Biol. 1415, 245-262 (2016). 
39. Langmead, B. Aligning short sequencing reads with Bowtie. Curr. Protoc. Bioinforma. Chapter 11, Unit 11, 17 (2010).

40. Love, M. I., Huber, W. \& Anders, S. Moderated estimation of fold change and dispersion for RNA-seq data with DESeq2. Genome Biol. 15, 550 (2014).

41. Heinz, S. et al. Simple combinations of lineage-determining transcription factors prime cis-regulatory elements required for macrophage and B cell identities. Mol. Cell 38, 576-589 (2010).

42. Braig, S. \& Bosserhoff, A. K. Death inducer-obliterator 1 (Dido1) is a BMP target gene and promotes BMP-induced melanoma progression. Oncogene $\mathbf{3 2}$, 837-848 (2013).

43. Dorn, C., Weiss, T. S., Heilmann, J. \& Hellerbrand, C. Xanthohumol, a prenylated chalcone derived from hops, inhibits proliferation, migration and interleukin-8 expression of hepatocellular carcinoma cells. Int. J. Oncol. 36 435-441 (2010).

44. Huang, D. W. et al. The DAVID Gene Functional Classification Tool: a novel biological module-centric algorithm to functionally analyze large gene lists. Genome Biol. 8, R183 (2007).

45. Huang, D. W. et al. DAVID Bioinformatics Resources: expanded annotation database and novel algorithms to better extract biology from large gene lists. Nucleic Acids Res. 35, W169-W175 (2007).
46. Szklarczyk, D. et al. STRINGV10: protein-protein interaction networks, integrated over the tree of life. Nucleic Acids Res. 43, D447-D452 (2015).

47. Szklarczyk, D. et al. The STRING database in 2017: quality-controlled protein-protein association networks, made broadly accessible. Nucleic Acids Res. 45, D362-D368 (2017).

48. Mootha, V. K. et al. PGC-1alpha-responsive genes involved in oxidative phosphorylation are coordinately downregulated in human diabetes. Nat. Genet. 34, 267-273 (2003).

49. Subramanian, A. et al. Gene set enrichment analysis: a knowledge-based approach for interpreting genome-wide expression profiles. Proc. Natl Acad. Sci. USA 102, 15545-15550 (2005).

50. McLean, C. Y. et al. GREAT improves functional interpretation of cis-regulatory regions. Nat. Biotechnol. 28, 495-501 (2010).

51. McLean, H. S., Carriker, C. \& Bordley, W. C. Good to Great: quality-improvement initiative increases and sustains pediatric health care worker hand hygiene compliance. Hosp. Pediatr. 7, 189-196 (2017). 